\title{
Method of Constructing a Line Secretive Exit the Aircraft at a Given Point of the Detection Area Surveillance Radar Based on the Spectrum Analysis of the Doppler Frequency of the Received Signal
}

\author{
Valeriy V. Zamaraev ${ }^{\text {a }}$, A.S. Kutuzova, \\ Igor V. Lyutikov*b and Dmitry V. Malcev \\ ${ }^{a}$ SIC Central Research Institute \\ of the Russian Defense Ministry VKO \\ Tver, Russian Federation \\ ${ }^{b}$ Siberian Federal University \\ Krasnoyarsk, Russian Federation \\ ${ }^{c}$ Military Academy of Aero-Space Defence \\ named after the Marshal of Soviet Union G.K. Zhukov \\ Tver, Russian Federation
}

Received 02.03.2021, received in revised form 22.03.2021, accepted 21.05.2021

\begin{abstract}
The article noted the contradictions in the theory and practice of the subject area of the study confirming the urgency of the task of constructing the trajectory of a hidden exit the aircraft at a given point of the detection area surveillance radar based on the spectrum analysis of the Doppler frequency of the received signal, the proposed initial data (initial conditions), describes the scientific and methodological apparatus of the embodiment of the method for generating control signals to implement the synthesized method, which allows to increase the effectiveness of air and space attack.
\end{abstract}

Keywords: aerospace defense, pulse-Doppler, detection of signal, frequency deviation, method for constructing a special flight path.

(C) Siberian Federal University. All rights reserved

This work is licensed under a Creative Commons Attribution-Non Commercial 4.0 International License (CC BY-NC 4.0).

* Corresponding author E-mail address: lyutikovigor@mail.ru 


\title{
Метод построения траектории скрытного выхода \\ летательного аппарата в заданную точку \\ зоны обнаружения обзорного радиолокатора, \\ основанный на анализе спектра доплеровских частот \\ принимаемого сигнала
}

\author{
В. В. Замараев ${ }^{\text {a }}$, А.С. Кутузов ${ }^{\text {, }}$ \\ И. В. Лютиков ${ }^{0}$, Д. В. Мальцев ${ }^{\text {в }}$ \\ ${ }^{a}$ НИЦ Центральный научно-исследовательский институт \\ Военно-воздушных сил Минобороны России \\ Российская Федерация, Тверь \\ ${ }^{6}$ Сибирский федеральный университет \\ Российская Федерация, Красноярск \\ ${ }^{8}$ Военная академия воздушно-космической оборонь \\ им. Маршала Советского Союза Г.К. Жукова \\ Российская Федераџия, Тверь
}

\begin{abstract}
Аннотация. В статье отмечены противоречия в теории и практике предметной области исследования, подтверждающие актуальность задачи построения траектории скрытого выхода летательного аппарата в заданную точку зоны обнаружения обзорного радиолокатора, основанного на анализе спектра доплеровских частот принимаемого сигнала, предложены исходные данные (начальные условия), описан научно-методический аппарат и вариант реализации способа выработки сигналов управления в интересах реализации синтезируемого метода, позволяющие повысить эффективность применения средств воздушно-космического нападения.
\end{abstract}

Ключевые слова: воздушно-космическая оборона, импульсно-доплеровский, обнаружение сигнала, девиация частоты, метод построения специальной траектории полета.

Цитирование: Замараев, В.В. Метод построения траектории скрытного выхода летательного аппарата в заданную точку зоны обнаружения обзорного радиолокатора, основанный на анализе спектра доплеровских частот принимаемого сигнала /В. В. Замараев, А. С. Кутузов, И. В. Лютиков, Д. В. Мальцев // Журн. Сиб. федер. ун-та. Техника и технологии, 2021, 14(3). C. 281-291. DOI: 10.17516/1999-494X-0309

\section{Введение}

В настоящее время все большая роль отводится беспилотным летательным аппаратам (БЛА), усложняются решаемые ими задачи. В частности, кроме выполнения непосредственной задачи, которая обычно выражается в целевой функции, зачастую требуется обеспечить выполнение ряда дополнительных условий. Одним из таких условий является обеспечение скрытности достижения заданного объекта. К настоящему времени проведено много работ по снижению радиолокационной заметности летательных аппаратов (ЛА) за счет уменьшения их эффективной поверхности рассеяния (ЭПР), разрабатываются специальные средства и методы преодоления средств обороны на малых высотах полета. Ответной мерой выступает размещение радиолокационных средств обнаружения (РЛС) на специальных вышках, при использовании которых появляется возможность создания сплошного радиолокационного поля на минимальных вы- 
сотах. Однако одним из предпочтительных путей скрытного полета ЛА к заданному объекту признано использование специальной траектории полета (СТП) ЛА. В основе построения СТП лежит анализ спектра доплеровских частот радиолокационного сигнала обзорного радиолокатора и принимаемого на борту ЛА [1,3-7].

\section{Исходные данные и научно-методический аппарат}

\section{для синтеза метода построения специальной траектории полета летательного аппарата}

Метод построения СТП ЛА в заданную точку в зоне обнаружения обзорного радиолокатора заключается в следующем.

Предполагаются известными следующие исходные данные:

положение ЛА относительно заданной точки (объекта О) в начальный момент времени $t_{0}$ в полярной системе координат $r_{0}=r\left(t_{0}\right)=R_{0} ; \beta_{0}=\beta\left(t_{0}\right)$;

скорость ЛА $v$;

допустимое ускорение ЛА $a_{\max }$;

на борту ЛА находится разведывательный приемник, информация которого позволяет оценить такие параметры радиолокатора, как темп обзора, текущее положение диаграммы направленности антенны (ДНА), а также некоторые параметры спектра, такие как доплеровская частота, частотная девиация.

В точке О находится радиолокатор (рис. 1), осуществляющий круговой обзор с перио-

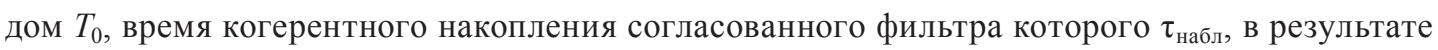
работы которого появляются стробы: временной $\left(-t_{i}-\Delta ; t_{i}+\Delta\right)$, соответствующий интервалу времени $\tau_{\text {набл}}$ в течение которого ЛА находится в главном лепестке ДНА радиолокатора, и спектральный - с шириной полосы $\Delta f$, параметры которого зависят от времени когерентного накопления.

В интересах обеспечения скрытного выхода ЛА в заданную точку задается такая траектория движения ЛА (за счет выбора параметров маневра и соответствующих им команд управления на исполнительные органы ЛА), при которой отраженный от ЛА сигнал в момент его облучения радиолокатором $t_{x}=t_{\mathrm{BC}}$ находится внутри спектрального строба с нулевой доплеровской частотой. Это условие должно выполняться при каждом попадании ДНА радиолокатора на ЛА.

Формально необходимо, чтобы одновременно были выполнены следующие условия:

$$
\begin{aligned}
& -\Delta f \leq f_{\text {д }}\left(t_{x}\right) \leq \Delta f ; \\
& \beta_{\text {изл }}\left(t_{x}\right)=\beta_{\text {ц }}\left(t_{x}\right) ; \\
& t_{k i-1}<t_{x i}<t_{k i-1}+T_{0},
\end{aligned}
$$

где $t_{k i-1}$ - время окончания облучения ЛА на $i$-м шаге; $\beta_{\text {изл }}\left(t_{x}\right)$ - азимут ДНА радиолокатора в момент облучения ЛА; $f_{\text {д }}\left(t_{x}\right)$ - доплеровская частота сигнала, отраженного от ЛА.

Для обеспечения скрытного полета ЛА в зоне обнаружения обзорного радиолокатора необходимо определить значения параметров управления как функций времени, т. е. найти траекторию полета ЛА при следовании к объекту.

Предположим, что скрытный полет ЛА в зоне обнаружения обзорного радиолокатора совершен в случае, если ЛА попадает из точки А в точку О (рис. 1).

$$
-283-
$$




$$
\mathrm{T}_{0}=\mathrm{T}_{\mathrm{p}}
$$

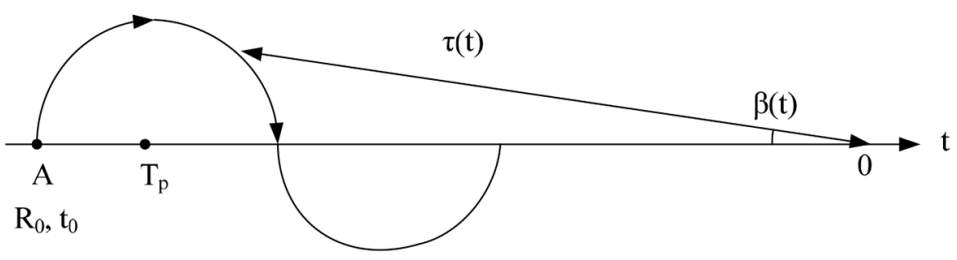

a)

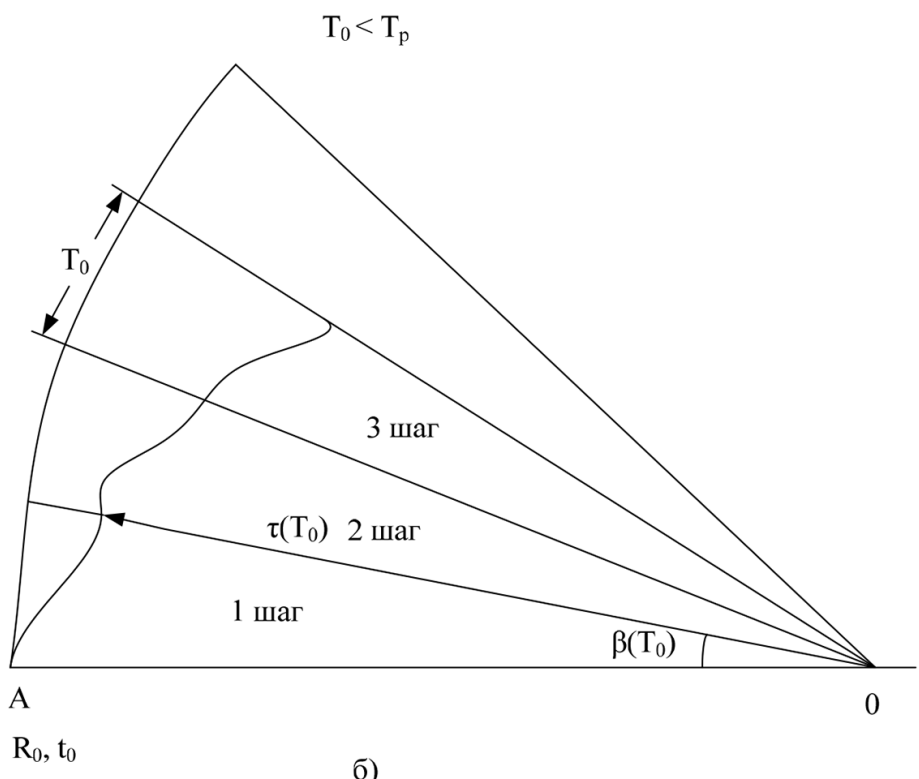

б)

Рис. 1. Начальный участок траекторий полета ЛА для различных условий

Fig. 1. The initial section of the aircraft flight paths for various conditions

В качестве показателя эффективности скрытного полета ЛА целесообразно выбрать интервал времени от момента входа ЛА в зону обнаружения обзорного радиолокатора до момента первого обнаружения ЛА.

Основными этапами решения задачи скрытного полета ЛА в заданную точку являются следующие.

На первом этапе осуществляется построение траектории полета ЛА по информации о периоде вращения ДНА РЛС, ее текущем положении в пространстве, о месте дислокации радиолокатора. При этом предполагается использовать априорные данные и данные, полученные на предыдущих интервалах наблюдения за режимом работы радиолокатора.

На втором этапе производится уточнение параметров траектории ЛА по текущей информации о доплеровском спектре принимаемого сигнала с учетом того, что в разведывательном приемнике ЛА происходит когерентное накопление сигнала. Для определенности принято, что время когерентного накопления равно времени облучения ЛА. 
На первом этапе построения СТП ЛА производится аналитический расчет времени «встречи» ДНА радиолокатора с ЛА $t_{B C}$, а также выбор параметров маневра, обеспечивающих указанную «встречу» с соблюдением требуемых условий.

Рассмотрим основные операции и соответствующие формульные соотношения, позволяющие их выполнить. Прежде всего отметим, что в связи с тем, что ДНА радиолокатора вращается с постоянной скоростью, весь интервал анализа траектории разбивается на участки длительностью, равной периоду вращения ДНА.

В зависимости от соотношения периода обзора $T_{0}$ пространства радиолокатором и времени разворота ЛА на 180 градусов $T_{p \text { max }}$ при аналитической оценке времени «встречи» ДНА радиолокатора с ЛА возможны две ситуации:

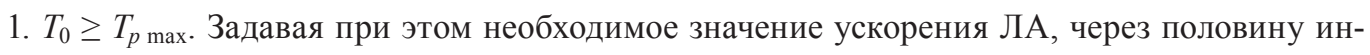
тервала времени разворота ЛА можно обеспечить равенство нулю радиальной составляющей скорости ЛА в точке его «встречи» с ДНА РЛС.

2. $T_{0}<T_{p}$. В этом случае ЛА не успевает развернуться на 180 градусов за период вращения антенны радиолокатора. Поэтому выполнение требуемого условия в момент «встречи» с ДНА можно выполнить в промежуточной точке (под другим азимутом), а для построения всей траектории применить многошаговую процедуру.

Результаты оценки времени выхода ЛА в заданную точку для различных значений периода обзора радиолокатора и ускорения ЛА приведены в табл. 1, 2.

Основные особенности построения специальной траектории полета ЛА при его движении из точки А в точку О заключаются в следующем (рис. 1 и 2).

Моменты «встречи» ДНА радиолокатора с ЛА представлены на рис. 1б. Предполагается, что в начальной точке выполнялись требуемые условия (1) и (2).

Следующая точка, в которую должен попасть ЛА, сдвинута по времени на величину $t_{B C}$, причем доплеровская частота сигнала, отраженного от ЛА в этой точке, должна быть равна 0 (рис. 2).

Для того чтобы попасть в эту точку, ЛА (не меняя модуля скорости) должен совершить двойной маневр курсом с максимально допустимым ускорением (первую половину интервала увеличивая скорость, а вторую уменьшая ее до нуля) (рис. 2б).

Таблица 1. $\left(r_{0}=20\right.$ км; $T_{0}=10$ сек $)$

Table 1. $\left(r_{0}=20 \mathrm{~km} ; T_{0}=10 \mathrm{sec}\right)$

\begin{tabular}{|c|c|c|c|}
\hline$a_{\text {max }}, \mathrm{M} /$ сек $^{2}$ & 30 & 50 & 100 \\
\hline$t_{\text {вbl } x}$, сек & 300 & 200 & 100 \\
\hline
\end{tabular}

Таблица 2. ( $r_{0}=20$ км; $T_{0}=2$ сек $)$

Table 2. $\left(r_{0}=20 \mathrm{~km} ; T_{0}=2 \mathrm{sec}\right)$

\begin{tabular}{|c|c|c|c|}
\hline$a_{\text {max }}, \mathrm{M} /$ сек $^{2}$ & 30 & 50 & 100 \\
\hline$t_{\text {вых }}$, сек & 1700 & 1000 & 500 \\
\hline
\end{tabular}




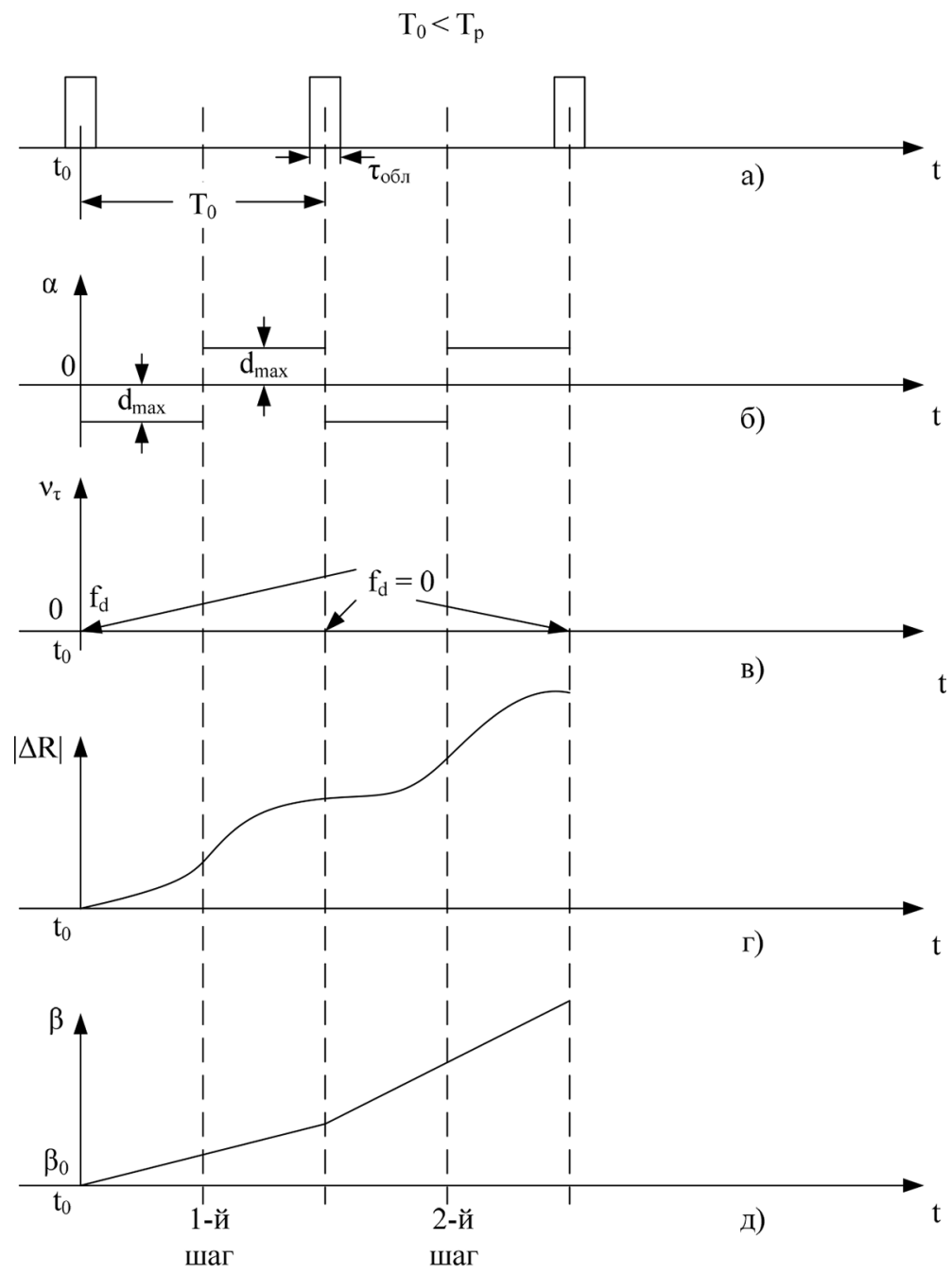

Рис. 2. Графики, иллюстрирующие пространственно-временную взаимосвязь процессов в системе «ЛА заданная точка»

Fig. 2. Graphs illustrating the spatial-temporal relationship of processes in the «aircraft-set point» system

Указанный процесс можно рассматривать как переход ЛА с орбиты $r=r_{0}$ на орбиту $r=r_{1}$.

Для осуществления указанного выше маневра необходимо выполнить следующие основные операции.

Начальные условия на первом шаге:

$$
r_{01}=r_{0} ; v_{r 01}=0 ; \beta_{01}=\beta_{0} ; v_{\beta 01}=\frac{v_{0}}{r_{0}} .
$$

1. Вычислить первую итерацию времени встречи, исходя из того, что ЛА и ДНА радиолокатора движутся равномерно по кругу:

$$
t_{B C}=\frac{2 \pi}{\frac{2 \pi}{T_{0}}-\frac{v}{r_{0}}} .
$$


2. Определить радиус разворота ЛА в исходной точке:

$$
r_{0 p}=\frac{v^{2}}{a_{\max }} .
$$

3. Найти координаты центра круга, по которому будет двигаться ЛА:

$$
x_{\mathrm{u} 1}=r_{0}-r_{p 1} ; y_{\mathrm{u} 1}=0 .
$$

4. Характер движения ЛА в прямоугольной системе координат (с заданными центром и радиусом) определить по выражениям

$$
x-x_{\mathrm{L} 1}=r_{0 p} \cos \omega t ; y-y_{\mathrm{L} 1}=r_{0 p} \sin \omega t,
$$

где $\omega$ - угловая частота вращательного движения ЛА.

5. Вычислить координаты ЛА на момент $\frac{t_{B C}}{2}: x\left(\frac{t_{B C}}{2}\right) ; y\left(\frac{t_{B C}}{2}\right)$ определить дальность и азимут ЛА (в системе координат радиолокатора) на момент $\frac{t_{B C}}{2}: r\left(\frac{t_{B C}}{2}\right) ; \beta\left(\frac{t_{B C}}{2}\right)$.

6. Определить изменение дальности и азимута за указанный временной интервал (рис. 2г, д):

$$
\Delta R_{1}=r_{0}-r\left(\frac{t_{B C}}{2}\right) ; \Delta \beta_{1}=\beta_{0}-\beta\left(\frac{t_{B C}}{2}\right) .
$$

7. В силу симметрии второго участка маневра на первом шаге прирост дальности и азимута будет вдвое выше и координаты ЛА в конце первого шага (начальные координаты второго шага) равны

$$
r_{k 1}=r_{0}-2 \Delta R_{1} ; \beta_{k 1}=\beta_{0}-2 \Delta \beta_{1}
$$

На основании координат ЛА уточняется его угловая скорость и производится вторая итерация закона движения ЛА на первом шаге.

Из анализа представленных рис. 1, 2 следует, что в конце каждого шага доплеровская частота равна нулю, что обеспечивает наибольшую скрытность отраженных от ЛА сигналов при любых технических параметрах радиолокатора (времени когерентного накопления, скорости вращения антенны).

При этом на каждом шаге происходит уменьшение расстояния между ЛА и радиолокатором. Если конечной точкой маршрута является радиолокатор, то процесс формирования СТП будет происходить до тех пор, пока координаты ЛА не попадут в заданную окрестность точки O. Если координаты конечной точки маршрута отличаются от точки О и равны $\left(R_{k}, \mathrm{~B}_{k}\right)$, то движение по указанной схеме будет происходить до тех пор, пока дальность ЛА до радиолокатора не сравняется с дальностью конечной точки маршрута $R_{k}$. После этого ЛА будет двигаться непрерывно по окружности, пока не достигнет точки $\mathrm{B}_{k}$.

При использовании предлагаемого метода построения СТП ЛА обеспечивается скрытный подход к заданной точке на всех этапах построения траектории. Однако при этом увеличивается время достижения указанной конечной точки траектории, что является «платой» за скрытность. По форме специальная траектория полета ЛА похожа на спираль.

$$
-287-
$$


Таблица 3

Table 3

\begin{tabular}{|c|c|c|c|c|}
\hline $\mathrm{R}$, Км & 20 & 10 & 5 & 1 \\
\hline Гц & 1 & 2 & 4 & 20 \\
\hline
\end{tabular}

Анализ факторов, влияющих на эффективность решения задачи скрытного полета ЛА в заданную точку, позволил выработать предложения по корректировке полученной СТП ЛА.

В частности, доплеровский спектр сигнала, отраженного от ЛА (за время его облучения) и принятого радиолокатором, не должен выходить за пределы полосы пропускания нулевого доплеровского фильтра. Предварительные результаты расчета доплеровского спектра частот отраженного от ЛА сигнала (табл. 3) подтверждают это утверждение.

Расчет выполнен в предположении, что длительность интервала облучения ЛА диаграммой направленности антенны радиолокатора определялась согласно выражению $\tau_{\text {обл }}=T_{0} \frac{\Delta \varphi}{2 \pi}$ ( $\Delta \varphi$ - ширина ДНА радиолокатора) и была равна $\tau_{\text {обл }}=0,01 \mathrm{c}$. При этом доплеровская частота отраженного сигнала равна нулю только в одной точке (в которой ЛА имеет нулевую радиальную скорость $v_{r}=0$ ), после этой точки она изменяется по линейному закону с девиацией

$$
\frac{d f}{d t}=\frac{v_{\tau}^{2}}{R_{i} \lambda}
$$

где $v_{\tau}$ - тангенциальная скорость ЛА (относительно радиолокатора); $\lambda$ - длина волны радиолокатора [3-7].

За время облучения ЛА общее изменение доплеровской частоты составит

$$
\Delta f=T \frac{\Delta \varphi v_{\tau}^{2}}{2 \pi R_{i} \lambda}
$$

Возможность выхода части отраженного сигнала из нулевого доплеровского фильтра в каждом конкретном случае оценивается подобным образом. И если такая возможность существует, то необходимо принять меры по сохранению примерно нулевой доплеровской частоты отраженного сигнала. Для этого траекторию ЛА следует скорректировать: в районе точки «встречи» с ДНА радиолокатора летательный аппарат необходимо вывести на определенное время на орбиту с нулевой доплеровской скоростью, т. е. придать ему ускорение, равное $a=v / R$.

В интересах корректировки траектории полета ЛА целесообразно рассматривать следующие временные интервалы:

интервал от начала маневра (на каждом шаге) до момента переключения направления движения $Y_{1}:-t_{\mu i}^{1} \leq t \leq t_{n i}^{1}$;

интервал от момента переключения направления движения до завершения маневра (на каждом шаге) $y_{2}:-t_{n i}^{1} \leq t \leq t_{\kappa i}^{1}$;

интервал полета в районе облучения ЛА радиолокатором (доплеровская частота близка к нулю) $\mathrm{У}_{3}$.

Каждому из перечисленных временных интервалов присущи свои особенности.

$$
-288-
$$


Так, для первого интервала $\mathrm{V}_{1}$ закон изменения доплеровской частоты отраженного сигнала определяется аналитически. Полученные измерения доплеровской частоты с интервалом (между отсчетами), равным времени когерентного накопления, сравниваются с априорными данными. Проверяется гипотеза о том, что заданная выборка порождена расчетной функцией $f_{a \partial}^{1}(t)$. В качестве критерия для проверки этой гипотезы может быть использован критерий Манна-Уитни [2]. Если значимость гипотезы достаточно велика, корректировку траектории можно не производить. В случае, если измерения лежат выше расчетной кривой, есть опасность, что после переключения ускорения ЛА к моменту «встречи» (с ДНА радиолокатора) не успеет достичь требуемого значения радиальной скорости, и поэтому необходима корректировка расчетной траектории (в основном за счет параметров маневра).

Для второго интервала $\mathrm{У}_{2}$ также проверяется гипотеза о том, что спектральная выборка порождена теоретической кривой для этого интервала. Как и для интервала $\mathrm{У}_{1}$, при необходимости принимается решение о надобности корректировки траектории ЛА (путем изменения ускорения).

В районе точки «встречи» с ДНА радиолокатора ЛА должен двигаться определенным образом. При этом возможны следующие ситуации.

1. ЛА вышел в расчетную точку раньше, чем туда «пришла» ДНА радиолокатора:

$$
\begin{aligned}
& v_{r}\left(t_{\text {хла }}\right)=0 ; \\
& \beta\left(t_{\text {хла }}\right)<\beta_{\text {изл }}\left(t_{\text {хла }}\right) .
\end{aligned}
$$

В этом случае ЛА должен перейти на орбиту, на которой $v_{r}\left(t_{\text {хла }}\right)=0$, и продолжать движение на ней до тех пор, пока ДНА радиолокатора не «минует» ЛА. На этом маневре будет завершен первый этап.

2. ЛА вышел в расчетную точку позже, чем туда «пришла» ДНА радиолокатора. Это означает, что до этого момента доплеровская частота была отлична от нуля (нарушались условия скрытности полета), что, естественно, нежелательно для ЛА. Поэтому при выработке сигналов управления необходимо сделать некоторое «упреждение» и тем самым обеспечить выполнение условия (3).

Возможный вариант реализации описанного способа выработки сигналов управления в интересах реализации метода скрытного выхода ЛА в требуемую точку представлен на рис. 3 .

Для этого на борту ЛА необходимо иметь разведывательный приемник, по информации которого оцениваются входные параметры для работы соответствующих алгоритмов (период вращения антенны и ее текущее положение, как это делается, например, в [3]). На основании полученных данных рассчитывается момент «встречи» ЛА с ДНА радиолокатора и вырабатываются соответствующие команды управления на исполнительные элементы ЛА. Команды управления корректируются по результатам оценки текущего положения ДНА радиолокатора.

\section{Заключение}

Таким образом, отмеченные в статье противоречия в теории и практике предметной области исследования подтверждают актуальность предлагаемого метода построения траектории выхода летательного аппарата в заданную точку зоны обнаружения обзорного радиолокатора,

$$
-289-
$$




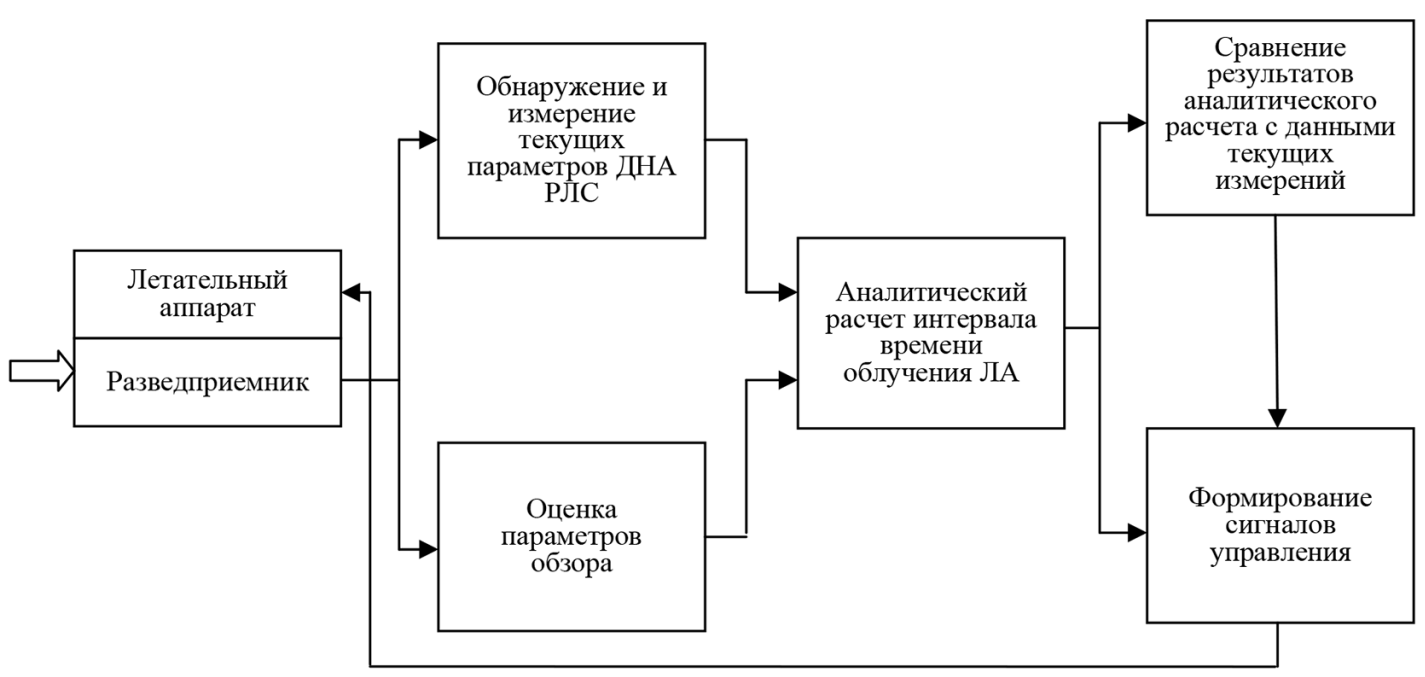

Рис. 3. Возможный вариант реализации способа выработки сигналов управления в интересах реализации метода скрытного выхода ЛА в требуемую точку

Fig. 3. A possible implementation of the method for generating control signals for the covert output of the aircraft to the desired point

основанного на анализе спектра доплеровских частот принимаемого сигнала. Предложенные исходные данные (начальные условия), научно-методический аппарат и вариант реализации способа выработки сигналов управления в интересах реализации синтезируемого метода позволят повысить характеристики скрытности и, как следствие, эффективность применения средств воздушного нападения, что необходимо подтвердить результатами имитационного и (или) натурного моделирования.

\section{Список литературы / References}

[1] Якунин А. С. История отечественной радиолокации. Под ред. А.С. Якунина. М.: Столичная энциклопедия, 2011. [Yakunin A. S. History of Russian radar. Ed. by A. S. Yakunin. M.: Stolichnaya encyclopedia, 2011 (in Russian)]

[2] Кендалл М. Дж., Стьюарт А. Статистические выводы и связи. М.: Наука, 1973. [Kendal M. J., Stuart A. Statistical conclusions and links. M., Science, 1973 (in Russian)]

[3] Ширман Я.Д., Багдасарян С. Т., Маляренко А. С., Леховицкий Д. И., Лещенко С.П., Лосев Ю.И., Николаев А.И., Горшков С.А., Москвитин С.В., Орленко В. М. Радиоэлектронные системы: Основы построения и теория. Москва: Радиотехника, 2007, 512 с. [Shirman Ya.D., Bagdasaryan S. T., Malyarenko A.S., Lexoviczkij D. I., Leshhenko S.P., Losev Yu. I., Nikolaev A.I., Gorshkov S. A., Moskvitin S. V., Orlenko V.M. Radioelectronic systems: bases of construction and theory. Moscow, Radiotekhnika, 2007, 512 p. (in Russian)]

[4] Замараев В. В., Шайдуров Г. Я., Лютиков И. В., Копылов В.А. Сеточный метод коллективного наведения и управляемого целераспределения группы ракет на основе анализа девиации частоты принимаемого головками самонаведения сигнала в интересах эффективной атаки групповой воздушной цели. Журнал Сибирского федерального университета. Техника и технологии, 2015, 8(8), 1103-1116. [Zamaraev V. V., Shajdurov G. Ya., Lyutikov I. V., Kopylov V. A. Grid 
method of collective guidance and controlled target distribution of a group of missiles based on the analysis of the deviation of the frequency of the homing signal received by the heads in the interests of an effective attack of the group air target, J. Sib. Fed. Univ. Eng. \& Technol., 2015, 8(8), 1103-1116 (in Russian)]

[5] Замараев В.В., Лютиков И.В. Метод защиты малоразмерных объектов от ракет на ближних рубежах в системе ВКО, использующий специальный измеритель дальности на основе анализа девиации частоты принимаемых сигналов. Журнал Сибирского федерального университета. Техника и технологии, 2019, 12(6), 719-723. [Zamaraev V. V., Lyutikov I. V. Method of Protecting Small Objects by Rockets on Near the Frontiers in Air and Space Defence System that Uses Special Measuring Range on the Basis of the Analysis the Frequency Deviation of the Received Signals, J. Sib. Fed. Univ. Eng. \& Technol., 2019, 12(6), 719-723 (in Russian)]

[6] Богданов А. В., Филонов А. А., Ковалев А. А., Кучин А.А., Лютиков И. В. Методы самонаведения истребителей и ракет класса «воздух-воздух» на групповую воздушную цель, Красноярск, Сиб. федер. ун-т, 2014, 168 с. [Bogdanov A. V., Filonov A. A., Kovalev A. A., Kuchin A. A., Lyutikov I. V. Methods of homing fighters and missiles «air-to-air» group air target, Krasnoyarsk, Sib. Fed. Univ., 2014, 168 p. (in Russian)]

[7] Богданов А. В., Закомолдин Д. В., Бондарев В. Н., Лютиков И. В. и др. Метод повышения помехозащищенности бортовой радиолокационной станции истребителя, Красноярск, Сиб. федер. ун-т, 2018, 182 с. [Bogdanov A. V., Zakomoldin D. V., Bondarev B. N., Lyutikov I. V. and another. Method of increasing the noise immunity of the fighter's onboard radar station, Krasnoyarsk, Sib. Fed. Univ., 2018, 182 p. (in Russian)] 